\author{
Veronica Lisi \\ Greta Garbo \\ Flavia Miccichè \\ Anna Stecca \\ Salvatore Terrazzino \\ Malida Franzoi \\ Elisa Tripoli \\ Maurizio Clementi \\ Alberta Leon \\ Pier Antonio Battistella
}

Published online: 20 July 2005

V. Lisi • F. Miccichè • A. Stecca

$\mathrm{S}$. Terrazzino $\bullet$ A. Leon

Research and Innovation (R\&I) Company,

Padua, Italy

V. Lisi • F. Miccichè • A. Stecca

S. Terrazzino $\bullet A$. Leon

Association "Antonio R. Cananzi"

for Headache and Cerebrovascular Diseases,

Padua, Italy

G. Garbo • M. Franzoi • E. Tripoli

M. Clementi • P.A. Battistella (《)

Department of Pediatrics,

University of Padua,

Via Giustiniani 3, I-35128 Padua, Italy

e-mail: battist@pediatria.unipd.it

Tel.: +39-049-8213505/3506

Fax: +39-049-8213509

\title{
Genetic risk factors in primary paediatric versus adult headache: complexities and problematics
}

\begin{abstract}
Numerous candidate genes for migraine have been proposed on the basis of their possible functional role in its pathogenesis. Genetic polymorphisms have been evaluated in association studies, some of which have been suggested to be susceptibility markers for adult migraine. To date, however, none of the identified polymorphisms in adult migraine susceptibility have been investigated in children, raising the possibility that they may not be necessarily involved in paediatric migraine susceptibility. This paper reviews studies of the genetic basis of migraine and summarises our experience in genetic association studies in primary paediatric headache susceptibility.
\end{abstract}

Key words Paediatric migraine • Susceptibility • Polymorphism • Endothelin type A receptor

\section{Introduction}

Recurring primary headaches, such as migraine or tension-type, are common during childhood (2.5\%) and adolescence (15\%) [1]. However, while ever increasing evidence shows that migraine is a complex neurovascular disorder with genetic factors playing a primary role in its aetiology, none of the genetic factors which have to date been shown to be linked to adult migraine susceptibility have been investigated in children, for whom primary headaches represent frequent causes for referral for neurologic assessment. In addition, while epidemiological, twin and family studies have revealed that approximately one-half of its variation is attributable to additive genes, with a negligible contribution of nonadditive genetic effects [2], the identification and validation of the underlying genetic risk factors poses enormous challenges even in adult migraine. The severity of migraine symptoms, such as the recurrence and duration of attacks and the age of onset, are variable among patients, thus rendering difficult both the definition of the appropriate phenotype as 
well as the selection of the best population in which to investigate the genetic load. Furthermore, some individuals may, independently of the presence or absence of environmental influences or type of prophylactic therapy undertaken, remain attack-free despite their genetic load, while others will continue to suffer.

Given all this, the aim of the present paper is to provide a short overview of the current status of genetic susceptibility studies in migraine, herein including the problematics involved in their application in paediatric $v s$. adult settings.

\section{Genetic factors contribute to migraine}

Genetic epidemiological studies of migraine show both positive family history and increased disease risk in relatives of migraine probands [3], the likelihood of which increases when the age at onset in the proband is below 20 years [4]. However, while familiarity supports the importance of genetic factors in migraine susceptibility, it does not prove heritability as it does not exclude the influences of shared environmental factors and common lifestyles. The significantly higher pairwise concordance rate among monozygotic compared to dizygotic twin pairs better supports the importance of genetic factors with hereditability estimates of about $50 \%$ for migraine without aura and with aura [5]. In the case of tension-type headache, the genetic factor may have a major role in the aetiopathogenesis of chronic tension-type headache, whereas environmental influence is stronger in episodic tension-type headache [6].

\section{Molecular studies of the genetic factors in adult migraine}

Genome-wide scanning approaches have identified migraine susceptibility loci on chromosomes 1, 4, 6, 11, 14, 19 and X [7]. By investigating familial hemiplegic migraine (FHM), a rare Mendelian form of migraine with aura transmitted by autosomal dominance [8], the identification of migraine genes such as the calcium channel gene CACNA1A [9] and the $\alpha 2$ subunit of the $\mathrm{Na}+\mathrm{K}+$ ATPase ATP1A2 gene $[10,11]$ was facilitated. In addition, given the major statistical power to detect several genes of small effect of the association studies by a candidate-gene approach, the relationship between migraine and candidate genes involved in pathogenic theories has been repeatedly investigated in adults [12]: unfortunately, the results have not often been replicated in subsequent, independent studies.

\section{Do the genes identified in the adult migraine susceptibil- ity explain susceptibility in children? Our experience}

Given the increased familial risk in relatives of migraine probands, we have, among the genes shown to be associated to adult migraine liability, recently focused on whether the $-231 \mathrm{G}>\mathrm{A}$ polymorphism in the endothelin 1 type $\mathrm{A}$ receptor (EDNRA) shown to strongly modulate the risk of adult migraine [13] contributes to paediatric migraine susceptibility. Results to date obtained, however, show that the $-231 \mathrm{G}>\mathrm{A}$ polymorphism in the EDNRA gene is neither associated with primary juvenile headache nor significantly correlated with main clinical features characteristic of the headache pathology, thus suggesting the possibility of an age-related interaction of the EDNRA polymorphic variant on migraine liability and disease expression [14].

Worth noting is that the only other published study tackling the genetic aspects of juvenile migraine has, based on evidence suggestive of an association between migraine and prothrombotic genetic risk factors, considered the factor $\mathrm{V}$ Leiden mutation [15] due to its high prevalence in patients with stroke and history of migraine [16]. As in our experience, no difference in the prevalence of this mutation was found in children and adolescents with migraine with aura $v s$. controls.

\section{Conclusive considerations}

As migraine is characterised by wide phenotypic and, most likely, genotypic heterogeneity, the identification and validation of the genetic risk factors involved critically depends on the accuracy of the determination of the disease phenotype. However, although the recently revised International Headache Society criteria (ICHD-II) have incorporated many developmentally related sensitive changes allowing for broader applicability in juvenile patients [17], the lack of specific clinical and biological markers reduce the possibility to differentially classify paediatric patients and, hence, the chance to identify the genetic risk factors involved. This complexity, in addition, further increases when considering the likelihood, of effects of "modifying" genes, as well as of co-morbidity, including phenotypical heterochronia, together with the possibility that the expression of the disease may vary as a function of age. Thus, whilst the heterogeneous complex traits of migraine may, in part, account for the current discrepancies in genetic susceptibility studies conducted in adult and paediatric migraineurs, other factors including comorbidity of migraine with other age-related disorders sharing common similar pathways may be involved [18, 19], while environmental and individually related factors may interact to raise the disease expression. 
In sum, although the identification and validation of the genetic risk factors in primary headache susceptibility introduce the possibility of identifying groups of patients who possess particular diagnostic or prognostic characteristics, it is particularly important to identify if and how these advances apply in different clinical settings, herein including the paediatric and adolescent settings, through the design of appropriate clinical trials. Only in this way it will be possible, at least on the basis of our attempts to identify genetic susceptibility markers in primary paediatric headache susceptibility based on those shown to be associated to adult migraine liability, to ensure that the resulting data are sufficiently robust in order to inform clinical decision making and to revise the available treatment strategies in primary headache disorders arising in either the paediatric or adult stage.

\section{References}

1. Bille B (1962) Migraine in school children. Acta Paediatr 51[Suppl 136]:1-151

2. Gervil M, Ulrich V, Kaprio J et al (1999) The relative role of genetic and environmental factors in migraine without aura. Neurology 53:995-999

3. Russell MB, Olesen J (1995) Increased familial risk and evidence of genetic factor in migraine. Br Med $\mathrm{J}$ 311:541-544

4. Baier WK (1985) Genetics of migraine and migraine accompagnee: a study of eighty-one children and their families. Neuropediatrics 16(2):84-91

5. Zviegler DK, Hur YM, Bouchard TJ et al (1998) Migraine in twins raised together and apart. Headache 38:417-422

6. Ulrich V, Gervil M, Olesen J (2004) The relative influence of environment and genes in episodic tension-type headache. Neurology 62:2065-2069

7. Kors EE, Vanmolkot KRJ, Haan J et al (2004) Recent findings in headache genetics. Curr Opin Neurol 17:283-288
8. Wessman M, Kaunisto MA, Kallela M et al (2004) The molecular genetics of migraine. Ann Med 36:462-473

9. Ophoff RA, Terwindt GM, Vergouwe MN et al (1996) Familial hemiplegic migraine and episodic ataxia type- 2 are caused by mutations in the $\mathrm{Ca} 2+$ channel gene CACNL1A4. Cell 87:543-552

10. De Fusco M, Marconi R, Silvestri L et al (2003) Haploinsufficiency of ATP1A2 encoding the $\mathrm{Na}+\mathrm{K}+$ pump alpha2 subunit associated with familial hemiplegic migraine type 2 . Nat Genet 33:192-196

11. Swoboda KJ, Kanavakis E, Xaidara A et al (2004) Alternating hemiplegia of childhood or familial hemiplegic migraine? A novel ATP1A2 mutation. Ann Neurol 55:884-887

12. Montagna $\mathrm{P}$, Cortelli $\mathrm{P}$, Mochi $\mathrm{P}$ (2003) The "typical" migraines: genetic studies and some practical considerations. J Headache Pain 4:47-56

13. Tzourio C, El Amrani M, Poirier O et al (2001) Association between migraine and endothelin type A receptor (ETA - 231 A/G) gene polymorphism. Neurology 56:1273-1277
14. Lisi V, Garbo G, Battistella PA et al (2005) Endothelin receptor A -231A/G polymorphism: lack of linkage to primary pediatric headache. Headache (submitted)

15. Soriani S, Borgna-Pignatti C, Trabetti E et al (1998) Frequency of factor V Leiden in juvenile migraine with aura. Headache 38:779-781

16. Kontula K, Ylikorkala A, Miettinen H et al (1995) Arg506Gln factor V mutation (factor $\mathrm{V}$ Leiden) in patients with ischaemic cerebrovascular disease and survivors of myocardial infarction. Thromb Haemost 73:558-560

17. Headache Classification Committee of the International Headache Society. (2004) The International Classification of Headache Disorders, 2nd edn. Cephalalgia 24:1-152

18. Tietjen GE (2000) The relationship of migraine and stroke. Neuroepidemiology 19:13-19

19. Lanzi G, Termine C, Rossi $M$ et al (2003) Are vascular disorders more prevalent in the relatives of children and adolescents with migraine? Cephalalgia 23:887-891 